\title{
Some Remarks on Uniform Convergence
}

\author{
By H. G. EggLeston \\ (Received 5th September 1949. \\ Read 5th May 1950.)
}

\section{Introduclion.}

A useful test for uniform convergence is that first established by Buchanan and Hildebrandt [4] which is as follows.

(A) "If a sequence $f_{n}(x)$ of monotonic functions converges to a continuous function $f(x)$ in $[a, b]$ then this convergence is uniform."

In $\$ 1$ of this paper it is shown that this test is included in a sequence of theorems, each of which establishes a type of uniform convergence. The first is a well-known topological theorem on limit sets, the second is a result on the limits of rectifiable arcs, the third is a generalisation of (A) due to Bebrend [3], the fourth is (A) itself, the fifth is a one-sided version of Bendixson's test and the sixth is. Bendixson's test.

In $\$ 2$ these theorems are partially extended to theorems of more than one variable.

A similar result to (A) in which the range $[a, b]$ is replaced by $(-\infty, \infty)$ has been established by Pólya [8] and extended by Conti [5]. There are corresponding extensions of the theorems proved here. The proof of Theorem 2 is related to the ideas used in papers by Radó and Reichelderfer [9], Ayer [1], Tsuji [11], and Ayer and Radó [2]. The paper by Goodstein [6] is also concerned with a theorem similar to (A).

(A) may be stated in the alternative form

"If a sequence of additive non-negative functions $F_{n}(x)$ defined for all measurable subsets of $[a, b]$ converges to an absolulely continuous limit function $F(x)$, then the convergence is uniform."

An example of the use of the alternative form of $(A)$ is given in Scheffé [10].

$$
\$ 1 \text {. }
$$

Let $S$ be a compact metric space and $\rho$ denote the distance function in $S$. For any subset $X$ of $S$ let $\mathcal{Z}(X, \epsilon)$ denote the set of all points whose distance from $X$ is less than $\epsilon, \epsilon$ being a positive number.

Theorem 1. Let $X_{n}$ be any sequence of sets in $S$; then for every $\epsilon>0$. there exists an integer $N$ such that for $n \geqq N$

$$
\mathcal{Z}\left(\widetilde{\lim } X_{n}, \epsilon\right) \supset X_{n}
$$

where $\overparen{\lim } X_{n}$ is the upper topological limit of $X_{n}$. 
For suppose that the contrary were the case. Then for some positive number $\epsilon_{0}$ there is a sequence of integers $n_{i}$ such that each $X_{n i}$ contains a point $x_{n_{i}}$ and

$$
\lim _{i \rightarrow \infty} n_{i}=\infty, \quad \rho\left(x_{n_{i}}, \overline{\lim } X_{n_{i}}\right) \geqq \epsilon_{0} .
$$

As $S$ is compact, a subsequence of the $x_{n i}$ tends to a point $p$. By (1)

$$
\rho\left(p, \overline{\lim } X_{n}\right) \geqq \epsilon_{0} .
$$

But by the definition of $\varlimsup i m X_{n}, p \subset \varlimsup \lim X_{n}$, a contradiction of (2)

THEOREM 2. Let $S$ be a bounded portion of Euclidean space of any (finite) number of dimensions and $X_{n}$ be a sequence of arcs in $S$. Suppose that the length of $X_{n}$ is $l_{n}$. If $\lim _{n \rightarrow \infty} l_{n}=l$ and there is an arc $X$ of length $l$ contained in lim $X_{n}$, then for every $\epsilon>0$ there exists an integer $N$ such

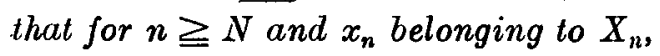

$$
\rho\left(x_{n}, X\right)<\epsilon \text {. }
$$

By Theorem 1 it is sufficient to show that

$$
X=\overline{\lim } X_{n} \text {. }
$$

If this is not the case then there is a point $y$ contained in $\varlimsup$ lim $X_{n}-X$. As $X$ is a closed set $\rho(y, X)=\eta>0$. There is a positive number $\delta$ such that $\eta>8 \delta$ and such that every polygonal line joining the two end-points of $X$, whose vertices belong to $X$ and whose segments are of length less than or equal to $\delta$, has total length greater than

$$
l-\eta / 4 \text {. }
$$

Such a polygonal line is constructed as follows. One end-point of the arc $X$, say $p_{0}$, is selected and called the first point, the other endpoint is called the last point $p^{\prime}$, and the other points are ordered in accordance with this nomenclature.

$p_{0}$ is the first point of the polygonal line. $p_{1}$ is $p^{\prime}$ if $\rho\left(p_{0}, p^{\prime}\right) \leqq \delta$, otherwise it is the last point after $p_{0}$ whose distance from $p_{0}$ is $\delta$.

Generally, $p_{i+1}$ is $p^{\prime}$ if $\rho\left(p_{i}, p^{\prime}\right) \leqq \cdot \delta$, otherwise it is the last point after $p_{i}$ for which $\rho\left(p_{i}, p_{i+1}\right)=\delta$. Let $p_{0} p_{1} \ldots p_{t-1} p^{\prime}$ be the polygonal line so formed and write $p^{\prime}=p_{t}$.

The circles $C\left(p_{i}, \delta / 2\right), i=0,1, \ldots, t$, of centre $p_{i}$ and radius $\delta / 2$, are non-overlapping and

$$
(t+1) \delta>l-\eta / 4
$$


As $p_{0}, p_{1}, \ldots, p_{t}$ belong to lim $X_{n}$, there is an integer $N_{0}$ such that

$$
\rho\left(p_{i}, X_{n}\right) \leqq \eta / 8(t+1), \quad i=0, \ldots, t, \quad n \geqq N_{0} .
$$

If the circle $C\left(p_{i}, \delta / 2\right)$ does not contain an end-point of $X_{n}$, the part of $X_{n}$ contained in it has length at least $\delta-\eta / 4(t+1)$. If it does contain an end-point the length contained is at least $\delta / 2-\eta / 8(t+1)$.

$X_{n}$ has two end-points; thus the set of circles $C\left(p_{i}, \delta / 2\right), i=0, \ldots, t$ contain a part of $X_{n}$ of length at least

$$
t(\delta-\eta / 4(t+1))>l-\eta / 2-\delta>l-5 \eta / 8 .
$$

Now $y \subset \varlimsup \lim X_{n}$, and thus for some integer $n$ arbitrarily large, say $n=m \geqq N_{0}$,

$$
\rho\left(y, X_{m}\right)<\eta / 4 \text {. }
$$

The circle $C(y, \eta-\delta / 2)$ does not overlap any of the $C\left(p_{i}, \delta / 2\right)$ and contains a part of $X_{n}$ of length greater than or equal to

$$
\eta-\delta / 2-\eta / 4>11 \eta / 16 \text {. }
$$

By (4), (5)

$$
l_{m} \geqq l+\eta / 16 .
$$

Since $m$ is arbitrarily large this is in contradiction with the fact that$\lim _{n \rightarrow \infty} l_{n}=l$.

CoRollary. Let $f_{n}(x)$ be a set of continuous functions defined over $a \leqq x \leqq b$ such that

(i) $\lim _{n \rightarrow \infty} f_{n}(x)=f(x), \quad a \leqq x \leqq b$,

(ii) $\lim _{n \rightarrow \infty}\left\{\lim _{h \rightarrow 0} \int_{a}^{b}\left[1+\left(\frac{f_{n}(x+h)-f_{n}(x)}{h}\right)^{2}\right]^{\frac{1}{b}} d x\right\}$

$$
=\lim _{h \rightarrow 0} \int_{a}^{b}\left[1+\left(\frac{f(x+h)-f(x)}{h}\right)^{2}\right] d x \text {. }
$$

(iii) $f(x)$ is continuous.

Then the convergence of the sequence $f_{n}(x)$ to $f(x)$ is uniform in $x, a \leqq x \leqq b$.

Denote by $X_{n}$ the set of points in the $(x, y)$ Euclidean plane with coordinates $\left(x, f_{n}(x)\right), a \leqq x \leqq b$, and by $X$ the set of points $(x, f(x)), a \leqq x \leqq b . \quad X_{n}$ and $X$ are arcs with lengths, say $l_{n}$ and $l$.

Condition (i) implies that $X \subset \lim X_{n}$ and condition (ii) that $\lim _{n \rightarrow \infty} l_{n}=l$. Thus by Theorem 2 for a given $\epsilon>0$,

$$
X_{n} \subset \mathcal{Z}(X, \epsilon) \quad n \geqq N=N(\epsilon) .
$$


For each point $p$ of $X$ form the segment whose midpoint is $p$ and which is parallel to the $y$-axis and is of length $\delta$. Let the set of points formed from all such segments be called $Y(X, \delta)$. Because $f(x)$ is continuous, for a given $\delta>0$ there is an $\epsilon>0$ such that

$$
\mathcal{U}_{1}(X, \epsilon) \subset Y(X, \delta)
$$

where $\mathcal{Z}_{1}(X, \epsilon)$ denotes that part of $\mathcal{Z}(X, \epsilon)$ lying in the closed strip $a \leqq x \leqq b$.

The conclusion now follows from (6) and (7).

Remark. The continuity condition on the $f_{n}(x), f(x)$ can be considerably relaxed: see e.g. Saks, Theory of the integral, p. 184.

Theorem 3. If $f_{n}(x), f(x)$ are monotonic in $[a, b]$ then $f_{n}(x) \rightarrow f(x)$ uniformily in $[a, b]$ if and only if (i) $f_{n}(x) \rightarrow f(x)$ for all $x$ of an everywhere dense set $E$ in $[a, b]$ containing the set $D$ of all discontinuities of $f(x)$, and

(ii) $f_{n}(x-0) \rightarrow f(x-0), \quad f_{n}(x+0) \rightarrow f(x+0)$ for all $x$ in $D$.

The necessity of the conditions is trivial. To prove their sufficiency the given conditions are used to reduce this theorem to the case of continuous $f(x)$ which is discussed in Theorem 4 .

Write the discontinuities of $f(x)$ as $c_{1}, c_{2}, \ldots$. Define the functions

$$
\begin{aligned}
& \phi(x)=\sum_{c_{k}<x}\left(f\left(c_{k}\right)-f\left(c_{k}-0\right)\right), \quad \phi_{n}(x)=\sum_{c_{k}<x}\left(f_{n}\left(c_{k}\right)-f_{n}\left(c_{k}-0\right)\right), \\
& \psi(x)=\sum_{c_{k}<x}^{\sum}\left(f\left(c_{k}+0\right)-f\left(c_{k}\right)\right), \quad \psi_{n}(x)=\sum_{c_{k}<x}^{\sum}\left(f_{n}\left(c_{k}+0\right)-f_{n}\left(c_{k}\right)\right), \\
& g(x)=f(x)-\phi(x)-\psi(x), \quad g_{n}(x)=f_{n}(x)-\phi_{n}(x)-\psi_{n}(x) .
\end{aligned}
$$

Then $g(x), g_{n}(x)$ are monotone and $g(x)$ is continuous. Conditions (i) and (ii) imply that

$$
\phi_{n}(x) \rightarrow \phi(x), \quad \psi_{n}(x) \rightarrow \psi(x)
$$

uniformly in $[a, b]$. Hence

$$
g_{n}(x) \rightarrow g(x)
$$

By the next theorem this convergence is uniform in $x$.

It follows that $f_{n}(x) \rightarrow f(x)$ uniformly in $x, a \leqq x \leqq b$.

Theorem 4. (Buchanan and Hildebrandt) If a sequence $f_{n}(x)$ of monotonic functions converges to a continuous function in $a \leqq x \leqq b$, say $f(x)$, then the convergence is uniform. 
An auxiliary sequence of functions $F_{i}(x)$ is defined as follows.

$$
F_{i}\left(x_{k}\right)=f\left(x_{k}\right) \quad \text { where } x_{k}=a+(b-a) k_{i} i, \quad k=0,1,2, \ldots i,
$$

and for other $x$ of $a \leqq x \leqq b, F_{i}(x)$ is defined by linear interpolation.

Then $F_{i}(x) \rightarrow f(x)$, uniformly by Theorem 2. Also

$$
\operatorname{Sup}_{a<x<b}\left|f_{n}(x)-F_{i}(x)\right| \leqq \operatorname{Max}_{k=0, \ldots, i-1}\left\{\left|f_{n}\left(x_{k}\right)-F_{i}\left(x_{k+1}\right)\right|+\left|f_{n}\left(x_{k+1}\right)-F_{i}\left(x_{k}\right)\right|\right\} \text {. }
$$

Thus $f_{n}(x) \rightarrow f(x)$ uniformly.

THEOREM 5. Let $f_{n}(x)$ be a sequence of continuous functions for each of which the upper right-hand derivative $f_{n}+(x)$ satisfies (i) $f_{n}+(x)>-\infty$ at every point $x, a \leqq x \leqq b$, except at most at those of an enumerable set, (ii) $f_{n}{ }^{+}(x) \geqq g(x)$ for almost all $x$, where $g(x)$ is Perron-integrable over $a \leqq x \leqq b$.

Suppose also that $f_{n}(x)$ tends to a continuous limit function $f(x)$. Then

$$
f_{n}(x) \rightarrow f(x) \text { uniformly in } x, a \leqq x \leqq b .
$$

Write $\phi_{n}(x)=f_{n}(x)-\int_{a}^{x} g(x) d x$ where the integral sign denotes the Perron integral. Conditions (i) and (ii) imply that,

$$
\phi_{n}(x+h)-\phi_{n}(x)=f_{n}(x+h)-f_{n}(x)-\int_{x}^{x+h} g(x) d x \geqq 0
$$

for $a \leqq x<x+h \leqq b$.

By Theorem 4, $\phi_{n}(x) \rightarrow f(x)-\int_{a}^{x} g(x) d x$ uniformly in $x$. Thus $f_{n}(x) \rightarrow f(x)$ uniformly in $x, a \leqq x \leqq b$.

Theorem 6. (Bendixson). If $f_{n}(x)$ is a sequence of differentiable functions in $a \leqq x \leqq b$ and $\left|f_{n}{ }^{\prime}(x)\right|<k$ for all $n$ and $x$, then if $f_{n}(x) \rightarrow f(x)$ as $n \rightarrow \infty$ it does so uniformly in $x, a \leqq x \leqq b$.

By Theorem 5 all that needs to be proved is that the limit function $f(x)$ is continuous. If this were not so there would be a point $x_{0}$ and a sequence of points $x_{1}, x_{2}, \ldots$ such that

$$
\lim _{i \rightarrow \infty} x_{i}=x_{0}, \lim _{i \rightarrow \infty} f\left(x_{i}\right) \neq f\left(x_{0}\right) .
$$

Thus there are two points $x_{0}, x_{k}$ such that

For $n$ sufficiently large

$$
\left|\left(f\left(x_{0}\right)-f\left(x_{k}\right)\right) /\left(x_{0}-x_{k}\right)\right|>K
$$

$$
\left|\left(f_{n}\left(x_{0}\right)-f_{n}\left(x_{k}\right)\right) /\left(x_{0}-x_{k}\right)\right|>K
$$


This is not possible because of the condition $\left|f_{n}{ }^{\prime}(x)\right|<K$.

Thus the theorem is established.

$\S 2$.

Of the preceding theorems, 2 and 3 do not extend directly to the case of functions of more than one variable. This is related to the fact that the behaviour of the areas of a sequence of surfaces is more complicated than that of the lengths of a sequence of arcs. The analogues of Theorems 4 and 6 are true but now require direct proofs. Similar direct proofs hold for Theorems 4 and 6 .

Theorem 7. Let $f_{n}\left(x_{1}, x_{2}, \ldots, x_{r}\right)$ be a sequence of functions of $r$ variables $x_{1}, x_{2}, \ldots, x_{r}$, defined over the ranges $a_{i} \leqq x_{i} \leqq b_{i}, i=1,2, \ldots, r$, such that each function is monotone in each of the $x_{2}, x_{3}, \ldots, x_{r}$ variables separately when all the other variables are fixed. Suppose also that the sequence converges to a continuous limit function $f\left(x_{1}, x_{2}, \ldots, x_{r}\right)$, and that this convergence is uniform in $x_{1}$ when $x_{2}, x_{3}, \ldots, x_{r}$ are kept fixed. Then the convergence is uniform in all the variables $x_{1}, x_{2}, \ldots, x_{r}$ simultaneously.

Since $f\left(x_{1}, x_{2}, \ldots, x_{r}\right)$ is continuous, for each $\epsilon>0$ there is an integer $N=N(\epsilon)$ such that

$$
\left|f\left(x_{1}{ }^{\prime}, x_{2}{ }^{\prime}, \ldots, x^{\prime}{ }_{r}\right)-f\left(x_{1}, x_{2}, \ldots, x_{r}\right)\right|<\epsilon
$$

provided $\left|x_{i}-x_{i}{ }^{\prime}\right|<1 / N, a_{i} \leqq x_{i} \leqq b_{i}, a_{i} \leqq x_{i}{ }^{\prime} \leqq b_{i}, i=1,2, \ldots, r$.

For each $\epsilon>0$, there is an integer $n_{0}(\epsilon)$ such that

$$
\begin{array}{r}
\mid f_{n}\left(x_{1}, a_{2}+\left(b_{2}-a_{2}\right) j_{2} / N, \ldots, a_{r}+\left(b_{r}-a_{r}\right) j_{r} / N\right) \\
-f\left(x_{1}, a_{2}+\left(b_{2}-a_{2}\right) j_{2} / N, \ldots, a_{r}+\left(b_{r}-a_{r}\right) j_{r} / N\right) \mid<\epsilon
\end{array}
$$

where $j_{2}, j_{3}, \ldots, j_{r}$ range over the integers $0,1, \ldots, N$ independently and $a_{1} \leqq x_{1} \leqq b_{1}, n \geqq n_{0}(\epsilon)$.

Consider now any point $\left(x_{1}, x_{2}, \ldots, x_{r}\right)$ where $a_{i} \leqq x_{i} \leqq b_{i}$, $i=1,2, \ldots, r$. There are two sets of integers $j_{2}, j_{3}, \ldots, j_{r}$, and $j_{2}{ }^{\prime}, j_{3}{ }^{\prime}, \ldots, j_{r}{ }^{\prime}$, such that

$$
\left.\begin{array}{l}
a_{i}+\left(b_{i}-a_{i}\right) j_{i} / N \leqq x_{i}<a_{i}+\left(b_{i}-a_{i}\right) j_{i}{ }^{\prime} / N \\
j^{\prime}{ }_{i}=j_{i}+1,0 \leqq j_{i}<N-1
\end{array}\right\} i=2,3, \ldots, r .
$$

Since the function is monotone

$$
f_{n}\left(x_{1}, y_{2}, \ldots, y_{r}\right) \geqq f_{n}\left(x_{1}, x_{2}, \ldots, x_{r}\right) \geqq f_{n}\left(x_{1}{ }^{\prime}, y_{2}{ }^{\prime}, \ldots, y_{r}{ }^{\prime}\right),
$$

where $y_{i}=a_{i}+\left(b_{i}-a_{i}\right) j_{i} / N$ or $y_{i}=a_{i}+\left(b_{i}-a_{i}\right) j_{i}^{\prime} / N$ and similarly for $y_{i}{ }^{\prime}$. (The particular value depends on whether the function is increasing or decreasing in the $i^{\text {th }}$ variable at the particular values of the other variables concerned and it may vary with $n$.) 
By (8) and (9), for $n \geqq n_{0}(\epsilon)$,

$$
\left|f_{n}\left(x_{1}, x_{2}, \ldots, x_{r}\right)-f\left(x_{1}, x_{2}, \ldots, x_{r}\right)\right|<2 \epsilon .
$$

This proves the theorem.

Remarks.

(i) This theorem contains the direct analogue of Theorem 4 because if the functions are monotonic in $x_{1}$ then the convergence with respect to $x_{1}$ is necessarily uniform.

(ii) This theorem also contains a more general result in which the condition on $f_{n}\left(x_{1}, x_{2}, \ldots, x_{r}\right)$ as a function of $x_{1}$ is that of the corollary to Theorem 2.

(iii) A similar conclusion to that of the theorem is true if the conditions on the sequence are "monotone in $r-s$ variables and uniformly convergent simultaneously in the other $s$ variables." This is not however true if the convergence is given to be uniform with respect to the $s$ variables separately.

\section{REFERENCES.}

[1] Ayer, M. C.

"On convergence in length," Proc. Nat. Acad. Sci. U.S.A., 31 (1945), 261-266.

,[2] Ayer, M. C. and Rado, T.

"A note on convergence in length," Bull. American Muth. Soc., 54 (1948), 533-539.

[3] Behrend, F. A.

"The uniform convergence of sequences of monotonic functions," J. Proc. Roy. Soc. New South Wales, 81 (1948), 167-168.

[4] Buchanan, H. E. and Hildebrandt, T. H.

"Note on the convergence of a sequence of functions of a certain type," Annals of Math. (2), 9 (1908), 123-126.

[5] Conti, $\mathbf{R}$.

"Estensione alle successioni di funzioni a variazione limitata di un criterio di PólyaCantelli per la convergenza uniforme su intervalli infiniti," Atti Accad. Naz. Lincei. Rend. Cl. Sci. Fis. Mat. Nat. (8), 4 (1948), 61-65.

[6] Goodstein, R. L.

"A theorem on uniform convergence," Muth. Gaz., 30 (1946), 287-290. 
[7] Marczewski, E. and Nosarzewska, M.

"Sur la convergence uniforme et la mesurabilité relative," Colloquizum Math., $1(1947), 15-18$.

[8] Pólya, G.

"Über den zentralen Grenzwertsatz der Wahrscheinlichkeitsrechnung und das Momentenproblem," Muth. Z., 8 (1920), 171-181.

[9] Radó, T. and Reichelderfer, P.

"Convergence in length and convergence in area," Duke Math. J., 9 (1942), 527.565.

[10] Scheffé, H.

"A useful convergence theorem for probability distributions," Ann. Math. Statistics, 18 (1947), 434-438.

[11] Tsuji, M.

"On Tonelli's theorems on a sequence of rectifiable curves," Japanese J. Math., 14 (1941), 401-410.

\section{University College,}

SWANSEA. 